Original article

Section: Food Chemistry

\title{
Exploring the Interactions Between Caffeic Acid and Human Serum Albumin Using Spectroscopic and Molecular Docking Techniques
}

\author{
Ali Jahanban-Esfahlan ${ }^{1,2}{ }^{\oplus}$, Leila Roufegarinejad ${ }^{3}$, Mahnaz Tabibiazar ${ }^{4,5}$, \\ José M. Lorenzo ${ }^{6,7} \odot$, Ryszard Amarowicz ${ }^{8 *}$ ( \\ ${ }^{1}$ Kidney Research Center, Tabriz University of Medical Sciences, Tabriz 5166-15731, Iran \\ ${ }^{2}$ Department of Biology, Faculty of Fundamental Sciences, University College of Nabi Akram (UCNA), Tabriz, Iran \\ ${ }^{3}$ Department of Food Sciences, Tabriz Branch, Islamic Azad University, Tabriz, Iran \\ ${ }^{4}$ Drug Applied Research Center, Tabriz University of Medical Sciences, Tabriz, Iran \\ ${ }^{5}$ Department of Food Science and Technology, Faculty of Nutrition and Food Science, \\ Tabriz University of Medical Sciences, Tabriz, Iran \\ ${ }^{6}$ Centro Tecnológico de la Carne de Galicia, Parque Tecnológico de Galicia, 32900 San Cibrao das Viñas, Spain \\ ${ }^{7}$ Área de Tecnología de los Alimentos, Facultad de Ciencias de Ourense, Universidad de Vigo, 32004 Ourense, Spain \\ ${ }^{8}$ Department of Chemical and Physical Properties of Food, Institute of Animal Reproduction and Food \\ Research of the Polish Academy of Sciences, Tuwima 10, 10-486 Olsztyn, Poland
}

Key words: human serum albumin (HSA), fluorescence, caffeic acid, interaction, molecular docking

Ultraviolet-visible (UV-Vis) and fluorescence spectroscopy along with molecular docking were used to explore the interaction between human serum albumin (HSA) and caffeic acid (CA). CA is one of the major representatives of hydroxycinnamic acids in plants and is commonly present in plant-based foods. The mechanism by which CA quenched HSA fluorescence was determined to be static, and the values obtained for thermodynamic parameters indicated that the CA and HSA interaction was spontaneous. Hydrogen bonds and van der Waals forces were the main driving forces stabilizing the complex. The binding constant was in the order of $10^{4} / \mathrm{M}$ and the number of binding sites for CA on HSA was calculated to be close to one. The results of fluorescence and UV-Vis spectroscopy showed that CA induced conformational changes in HSA structure. The distance of CA and the tryptophan residue of HSA, was determined to be $\sim 2 \mathrm{~nm}$ by using Forster resonance energy transfer theory. The mode of binding and the binding site of CA on albumin were examined by performing molecular docking calculations. CA interacted with albumin in subdomain IA, and non-covalent interactions stabilized the complex. CA showed a high affinity for albumin, and thus this phenolic compound would be distributed in the body upon interacting with HSA.

\section{ABBREVIATIONS}

CA - caffeic acid; FRET - Forster resonance energy transfer; H-bonds - hydrogen bonds; HCAs - hydroxycinnamic acids; HAS - human serum albumin; IFE - internal filter effect; SV - Stern-Volmer; UV-Vis - ultraviolet-visible; and vdW - van der Waals.

\section{INTRODUCTION}

Caffeic acid (CA) with the chemical name of 3,4-dihydroxycinnamic acid is a natural, plant-derived phenolic compound that belongs to the class of hydroxycinnamic acids (HCAs). CA is commonly present in foods of plant origin, with fruits and products obtained from them being a particu-

\footnotetext{
* Corresponding Author:

E-mail: r.amarowicz@pan.olsztyn.pl (R. Amarowicz)
}

larly abundant source of this compound [El-Seedi et al., 2012; Pirjo et al., 2006; Tomašević et al., 2019]. In fruits, it may constitute up to $70 \%$ of the total HCAs content [Sova \& Saso, 2020]. The relatively good bioavailability of CA makes it possible to interact with human serum albumin (HSA) in the body [Rashmi \& Negi, 2020]. In a cross-over study with 4 female and 3 male healthy ileostomy subjects, 95\% of the ingested caffeic acid was absorbed from the small intestine in humans [Olthof et al., 2001]. CA possesses different biological activities, such as antioxidant properties due to its high radical scavenging activity, and antimutagenic, anti-inflammatory, antidepressant, antimetastatic, anticarcinogenic, HIV replication-inhibitory, and antianxiety activities [Chen \& Ho, 1997; El-Seedi et al., 2012; Sova \& Saso, 2020]. Additionally, CA has been reported to induce apoptosis in cancerous cells and inhibit tumor proliferation in animal models CA [Bhat et al., 2007; Chung et al., 2006].

Among the various serum proteins in the bloodstream of the human body, HSA is the abundant biomacro- 
molecule $(\sim 60 \%)$. Its role in the circulatory system is crucial because it functions as a transporter for various chemicals and pharmaceuticals [Jahanban-Esfahlan et al., 2016, 2019]. Immediately after transport into the blood, various bioactive substances interact with albumin and are efficiently distributed throughout the body [Roufegarinejad et al., 2019]. Thus, HSA has a high affinity for a broad spectrum of endogenous- and exogenous molecules, including foodderived bioactive compounds or other various chemicals. Furthermore, the solubility of poorly-soluble compounds increased upon their binding to HSA and, thus, the weak or strong interactions between chemicals and albumin will affect their fate in the blood [Roufegarinejad et al., 2019]. HSA is categorized as a globular protein, and its molecular weight is $66.4 \mathrm{kDa}$. It has a heart-shaped structure, and includes 586 amino acids. Structurally, HSA includes three main homologous domains called domains I, II and III, and two A and B subdomains constructing each domain. Albumin contains two major binding sites named Sudlow's sites I and II. The first site is located in subdomain IIA, while the another is located in subdomain IIIA. Most of the molecules and chemicals bind to these regions of albumin [Jahanban-Esfahlan et al., 2015, 2019, 2020].

Although some data are available in the scientific literature for the interaction of $\mathrm{CA}$ and serum albumins [Adzet et al., 1988; Li et al., 2010; Min et al., 2004; Precupas et al., 2017; Sinisi et al., 2015; Skrt et al., 2012; Suryaprakash et al., 2000; Zhang et al., 2008], detailed information about the interaction of CA and HSA is scarce. Additionally, most of these investigations did not report full details regarding the binding of CA to albumin using fluorescence spectroscopy. Thus, the current study may be the first comprehensive and detailed report of the interaction of HSA and CA utilizing fluorescence, and UV-Vis spectroscopy approaches. Furthermore, molecular docking was performed using the ArgusLab software. The obtained results have been discussed to clarify the nature of the interaction between CA and HSA. The results of the present study are expected to fill the current gap in the protein binding properties of $\mathrm{CA}$, which is an important plant-based phenolic compound in human health and nutrition.

\section{MATERIALS AND METHODS}

\section{Materials}

CA and fatty acid-free HSA were obtained from Sigma-Aldrich (Saint Louis, MO, USA) and used as received. The analytical-grade solvents and reagents were used without additional purification in the present study. Double distilled water was used in all the experiments.

\section{Preparation of stock solutions}

The phosphate buffer considered in this study was prepared at a concentration of $10 \mathrm{mM}$ using potassium salts including $\mathrm{KH}_{2} \mathrm{PO}_{4}$ and $\mathrm{K}_{2} \mathrm{HPO}_{4}$ and then, $\mathrm{NaOH}$ was used for its $\mathrm{pH}$ adjustment. In the next step, phosphate buffer with physiological $\mathrm{pH}$ adjusted at 7.4 was used for the preparation of HSA stock and working solutions. Thus, the HSA stock solution was prepared by directly dissolving the protein powder in the prepared phosphate buffer. An ethanolic stock solution of CA with a concentration of $10 \mathrm{mM}$ was prepared by dissolving specific amounts of CA powder in ethanol. The solutions used for fluorescence and UV-Vis spectroscopy were diluted appropriately from the prepared stock solutions.

\section{Fluorescence spectroscopy}

A Jasco FP-750 fluorescence spectrophotometer (Kyoto, Japan) was used to record fluorescence spectra. The light source of the apparatus was a xenon lamp, and the width of the quartz cell was $1 \mathrm{~cm}$. An instrument composed of a stirrer and a cell holder with a water jacket was used as a temperature controller. The fluorescence spectroscopy experiments were carried out at temperatures of 290 , 300 and $310 \mathrm{~K}$. The corresponding fluorescence intensities for the emission wavelength (349 $\mathrm{nm}$ ) were obtained using an excitation wavelength of $290 \mathrm{~nm}$. A fixed slit width of $5 \mathrm{~nm}$ was applied for both excitation and emission wavelengths. The scan speed was $1200 \mathrm{~nm} / \mathrm{min}$. Using $2.5 \mu \mathrm{M}$ HSA (a constant concentration) and increasing concentrations of CA $(0$, $2.5,10,30,50,70$, and $90 \mu \mathrm{M}$ ), all fluorescence spectra were recorded at $\mathrm{pH}$ 7.4.

An experimental internal filter effect (IFE) may have reduced the emission intensity to some extent when spectra were recorded in the presence of increasing CA concentrations. This effect is an obvious issue affecting many fluorimetric methods, leading to a deviation of the results from the initial linearity, and therefore this effect must be considered. The fluorescence intensities were subsequently corrected for the absorption of the light at the excitation wavelength and reabsorption of the emitted light to reduce the IFE using the following equation:

$F_{c o r}=F_{o b s} 10^{\left(A_{e x}+A_{e m}\right) / 2}$

where: $\mathrm{F}_{\mathrm{cor}}$ and $\mathrm{F}_{\mathrm{obs}}$ are the corrected and observed fluorescence intensities, respectively, and $\mathrm{A}_{\mathrm{ex}}$ and $\mathrm{A}_{\mathrm{em}}$ are the absorption of the $\mathrm{CA}$ at the excitation and the emission wavelengths [Roufegarinejad et al., 2019], respectively. The fluorescence intensity reported in this study is the fluorescence intensity that has been corrected.

The data obtained from the HSA fluorescence quenching experiment were evaluated using the Stern-Volmer (SV) equation to clarify the mechanism by which CA quenched HSA. The SV equation is presented as follows [Lakowicz, 2006]:

$$
\begin{aligned}
& \frac{F_{0}}{F}=1+K_{S V}[Q]=1+K_{q} \tau_{0}[Q] \\
& K_{q}=K_{S V} / \tau_{0}
\end{aligned}
$$

where: $\mathrm{F}$ and $\mathrm{F}_{0}$ are the fluorescence emission intensities of HSA in the presence and absence of the quencher, respectively; $\mathrm{K}_{\mathrm{q}}$ is the constant of the quenching rate for the biomolecule; $\tau_{0}$ (equals $10^{-8} \mathrm{~s}$ ) is the average lifetime of the biomolecule in the absence of quencher [Chen et al., 1990; Eftink, 1991]; $[\mathrm{Q}]$ and $\mathrm{K}_{\mathrm{SV}}$ are the concentration of quencher and the con- 
stant of SV quenching, respectively. Linear plots of $\mathrm{F}_{0} / \mathrm{F} v s$. [Q] are only expected for the static quenching mechanism.

Another SV equation was also used in this study for a further analysis of the fluorescence quenching process [Lehrer, 1971]:

$\frac{F_{0}}{\left(F_{0}-F\right)}=\frac{1}{f_{a}}+\frac{1}{f_{a} K_{a}[Q]}$

where: $\mathrm{K}_{\mathrm{a}}$ and $\mathrm{f}_{\mathrm{a}}$ are defined as effective quenching constant for the available fluorophores and the fraction of the nearby fluorophores, respectively.

The modified logarithmic SV equation was used to calculate the binding constant $\left(\mathrm{K}_{\mathrm{b}}\right)$ and the number of binding sites (n) when ligands show an affinity to bind individually to similar binding sites on a biomolecule. The equation is presented as follows [Belatik et al., 2012; Ulrich, 1990]:

$\log \frac{\left(F_{0}-F\right)}{F}=\log K_{b}+n \log [Q]$

For the determination of $\mathrm{n}$ and $\mathrm{K}_{\mathrm{b}}$ values, the regression curves of $\log \left[\left(\mathrm{F}_{0}-\mathrm{F}\right) / \mathrm{F}\right]$ vs. $\log [\mathrm{Q}]$ were plotted. The $y$-coordinate and the slope of the obtained plots are $\mathrm{n}$ and $\mathrm{K}_{\mathrm{b}}$, respectively.

All the fluorescence experiments were performed in triplicate, and results were presented as the means $(n=3)$.

\section{UV-Vis spectroscopy}

The UV-Vis spectra of HSA were obtained using T70 UV/Vis spectrophotometer (PG Instrument Ltd, Lutterworth, $\mathrm{UK}$ ) in the presence and absence of CA at room temperature. The concentration of the protein was $20 \mu \mathrm{M}$, while four different concentrations of $\mathrm{CA}(0,20,50$, and $100 \mu \mathrm{M})$ were analyzed. The range of recorded UV-Vis spectra was 200-430 nm.

\section{Thermodynamic parameters}

Changes in both entropy $(\Delta S)$ and enthalpy $(\Delta H)$ were obtained from the van't Hoff equation (Eq. 6) by assuming the critical point that the change in enthalpy is negligible at the studied temperature range.

$\ln K=-\left(\frac{\Delta H}{R T}\right)+\left(\frac{\Delta S}{R}\right)$

$\Delta G=\Delta H-T \Delta S=-R T \ln K$

In Eq. 6, $\mathrm{K}$ is the constant for the effective quenching and it corresponds to the $\mathrm{K}_{\mathrm{a}}$ values obtained at the considered temperatures and $\mathrm{R}$ is the gas constant. The slope and the intercept of the plotted curves of $\ln \mathrm{K} v$. $1 / \mathrm{T}$ were used to determine the values of $\Delta \mathrm{S}$ and $\Delta \mathrm{H}$. The values of Gibb's free energy $(\Delta \mathrm{G})$ were obtained from Eq. 7 .

\section{Energy transfer studies}

The overlapping region of the UV-Vis spectrum for the CA molecule and the HSA fluorescence spectrum was considered to determine the energy transfer and the distance (r) between CA as the acceptor and the tryptophan (Trp) residue of HSA as the donor. An equal concentration of $30 \mu \mathrm{M}$ was used to obtain the UV-Vis spectrum of CA and HSA fluorescence spectrum, at wavelengths ranging from 200 to $500 \mathrm{~nm}$.

According to the theory of energy transfer presented by Forster, the value of $\mathrm{E}$ (the efficiency of energy transfer) was obtained from the following equation [Lakowicz, 2006]:

$E=1-\frac{F}{F_{0}}=\frac{R_{0}^{6}}{R_{0}^{6}+r^{6}}$

where: $\mathrm{R}_{0}$ is the critical distance with $50 \%$ energy transfer efficiency; $\mathrm{r}$ is the distance between the donor and acceptor molecules; and $\mathrm{F}$ and $\mathrm{F}_{0}$ are the fluorescence intensities of the HSA in the absence and presence of CA, respectively. $\mathrm{R}_{0}$ was obtained from Eq. 9:

$R_{0}^{6}=8.8 \times 10^{-25} k^{2} N^{-4} \Phi J$

where: $\mathrm{J}$ is the overlap between the integral of acceptor absorption and the emission spectrum of donor fluorescence, $\Phi$ is the quantum yield of the donor fluorescence, $\mathrm{N}$ is the medium refractive index, and $\mathrm{k}_{2}$ is the dipole spatial orientation factor [Lakowicz, 2006]. Accordingly, J was calculated by Eq. 10:

$J=\frac{\sum F(\lambda) \varepsilon(\lambda) \lambda^{4} \Delta \lambda}{\sum F(\lambda) \Delta \lambda}$

The molar absorption coefficient of the acceptor is $\varepsilon(\lambda)$ at wavelength $\lambda$, and the fluorescence intensity of the fluorescent donor at wavelength $\lambda$ is $F(\lambda)$. Notably, 2/3, 0.118, and 1.336 are the values proposed for $\mathrm{K}_{2}, \Phi$, and $\mathrm{N}$, respectively [Samari et al., 2012].

\section{Molecular docking studies}

The binding sites for CA molecules and the binding energy of the formed CA-HSA complex were investigated using molecular docking. The crystal structure of HSA (PDB ID: 1AO6) was obtained from the Worldwide Protein Data Bank [wwPDB, https://www.rcsb.org/structure/1ao6]. Upon the removal of water and ligand molecules and the addition of hydrogen atoms, the docking calculations were performed using the ArgusLab 4.0.1 docking software [Jahanban-Esfahlan et al., 2017]. Notably, 0.4 and $80 \times 80 \times 80$ angstroms were selected as the grid resolution and the size of the binding site bounding box, respectively. For all docking runs, a maximum of 200 candidate poses were used, and the docking engine was Argus Dock. A flexible form of the ligand, was selected, and the conformations were ranked to estimate the value of the binding energy using the Ascore scoring function. Ligand-receptor complexes resulting from molecular docking were comprehensively analyzed using PyMOL [Wang et al., 2008].

\section{RESULTS AND DISCUSSION}

\section{Quenching of HSA fluorescence in the presence of CA}

The fluorescence properties of aromatic amino acids, such as Trp, phenylalanine (Phe) and tyrosine (Tyr), in the structure of serum albumins enable researches to study the inter- 
A

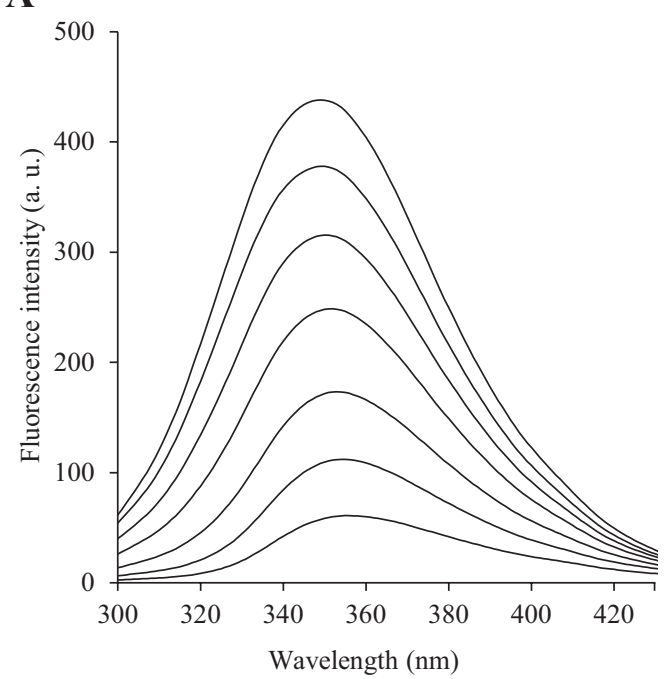

C

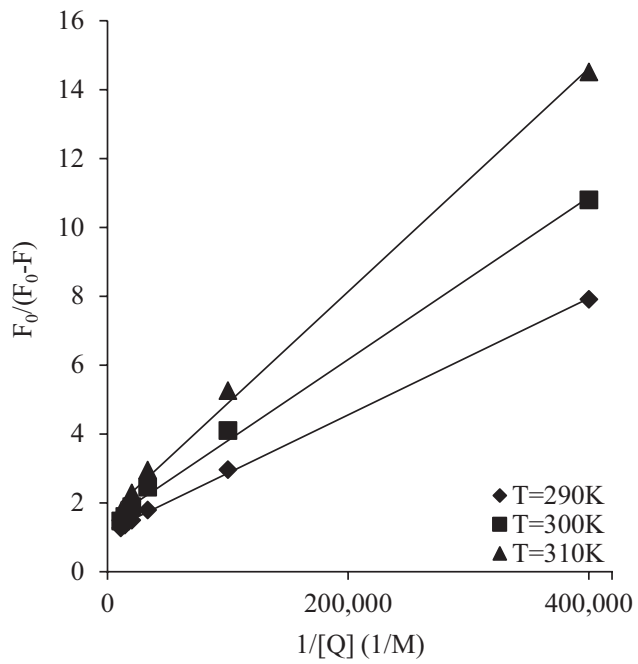

E

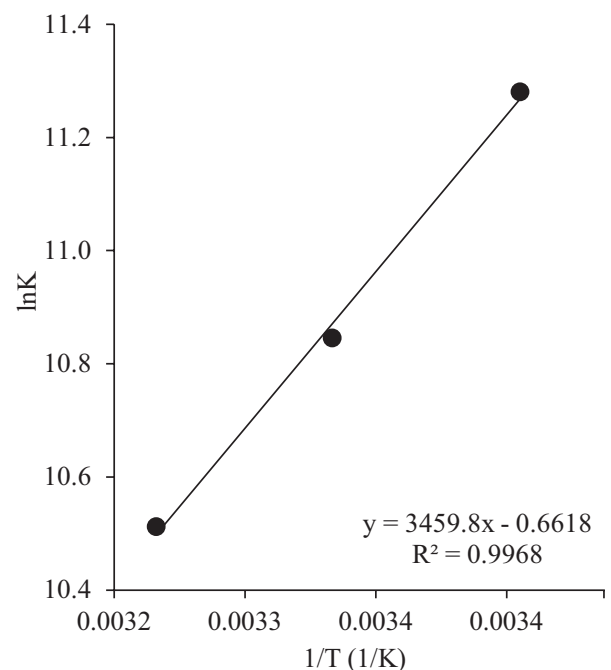

B

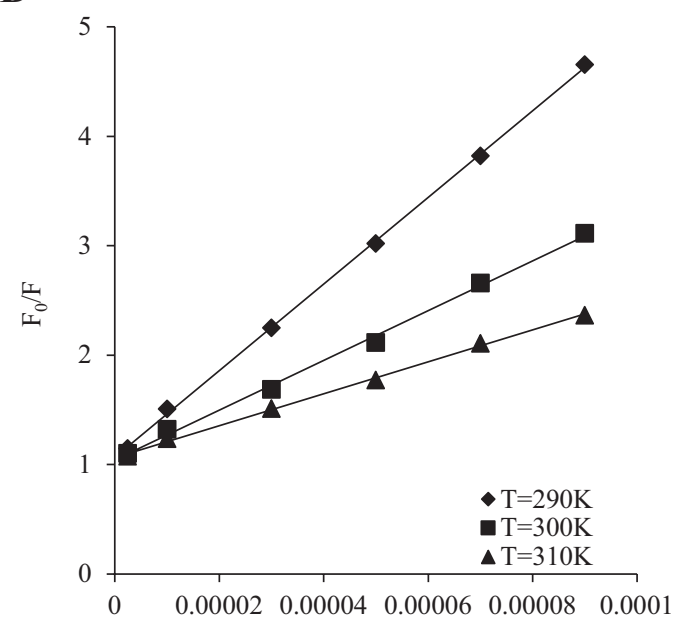

[Q] (M)

D

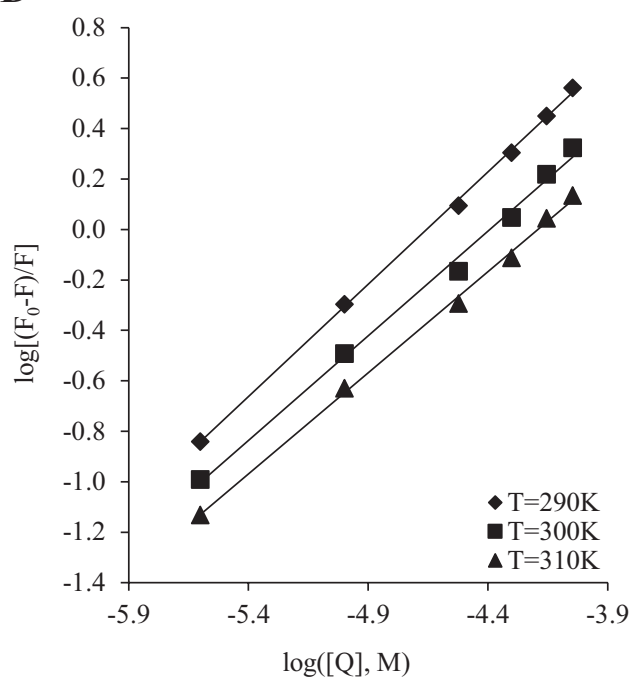

F

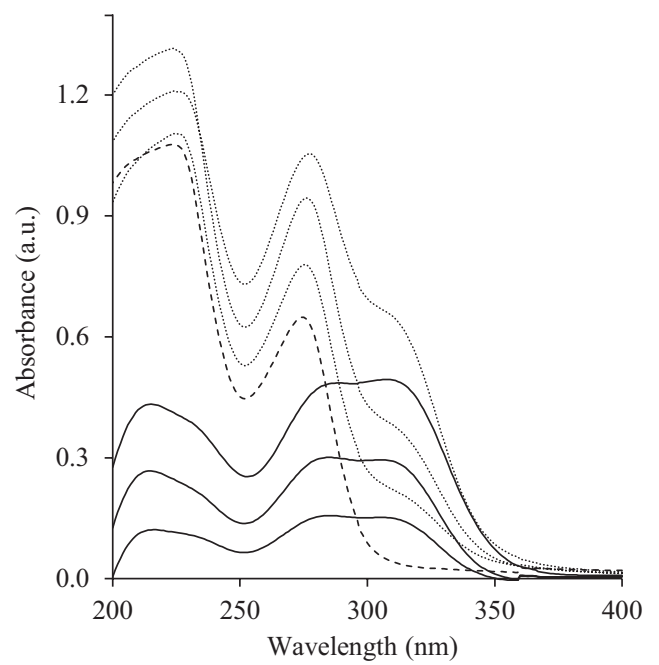

FIGURE 1. Fluorescence emission spectra of $2.5 \mu \mathrm{M}$ human serum albumin (HSA) mixed with different concentrations of caffeic acid (CA) $\left(\lambda_{\mathrm{ex}}=290 \mathrm{~nm}\right)$ at $310 \pm 1 \mathrm{~K}$. From top to bottom, CA concentrations were $0,2.5,10,30,50,70$, and $90 \mu \mathrm{M}$ (A). The Stern-Volmer (SV) plots obtained at different temperatures for the fluorescence quenching of HSA by CA (B). Modified SV plots obtained at different temperatures for the CA-HSA complex (C). Logarithmic SV plots used to determine the number of biding sites for CA molecules per HSA molecule (D). The Van't Hoff plot obtained for HSA-CA complex in a phosphate buffer and $\mathrm{pH} 7.4$ used to calculate the thermodynamic parameters (E). UV absorption spectra of HSA in the absence and presence of CA. The solid lines are the spectra of CA in the absence of HSA. The dashed line is the UV absorption spectrum of HSA alone. The dotted lines are HSA spectra in the presence of CA (F).UV-Vis absorption spectra of HSA in the absence spectrum of HSA alone, and the dotted lines represent the spectrum of HSA in the presence of CA (F). 
actions between different ligands and HSA using a fluorescence quenching method. For the HSA molecule, its intrinsic fluorescence is related to the Trp residue because of the low quantum yield of Phe or the ionization of Tyr [Mrkalic et al., 2021; Sudhamalla et al., 2010]. Additionally, the fluorescence of Tyr is generally quenched when it is located in the vicinity of a carboxyl or amino groups, as well as other Trp residue [Jahanban-Esfahlan et al., 2017]. Figure 1A shows the fluorescence emission spectra recorded for HSA before and after the addition of different concentrations of CA. The sharp peak near $350 \mathrm{~nm}$ was related to the fluorescence emission of HSA. A regular reduction in the fluorescence intensity from the top to the bottom of the recorded peaks was observed in the presence of increasing CA concentrations, conforming the binding of CA to HSA and the quenching of the intrinsic fluorescence intensity of albumin.

Furthermore, upon CA addition, the maximum peak in the obtained HSA emission spectra exhibited a remarkable shift from $348 \mathrm{~nm}$ to $357 \mathrm{~nm}(9 \mathrm{~nm})$, indicating a change in the polarity of the microenvironment surrounding the chromophore of HSA [Nair, 2018]. Similar results have been previously reported [Belatik et al., 2012; Cui at al., 2004; Mrkalić et al., 2021; Razzak et al., 2019]. This alteration is attributed to the loss of the compact albumin structure in the hydrophobic binding site pocket of subdomain IIA, and the location of Trp residue in this region of the protein is the reason of that alteration [Sułkowska 2002]. In the next steps, the obtained data for fluorescence quenching at a maximum peak of $349 \mathrm{~nm}$ and a temperature of $310 \pm 1 \mathrm{~K}$ were used for the quantitative analysis of CA and HSA interaction.

\section{Fluorescence quenching mechanism analysis}

Fluorescence quenching is caused by a reduction in the fluorescence quantum yield resulting from the interactions between the fluorophore and quencher molecules. Commonly, static and dynamic quenching are the two main mechanisms investigated when analyzing the type of fluorescence quenching. Usually, the formation of a ground-state complex without any fluorescence is recognized as static quenching, but a collisional encounter of the fluorophore and quencher is acknowledged as dynamic quenching [Lakowicz, 2006].

As illustrated in Figure 1B, the presented plots derived from the SV equation (2) for HSA in the presence of increasing concentrations of $\mathrm{CA}$ at three considered temperatures were linear. Thus, a static quenching mechanism underlies the HSA-CA interaction. Using the slope regression curve of $\mathrm{F}_{0} / \mathrm{F}$ vs. [Q], $\mathrm{K}_{\mathrm{q}}$ and $\mathrm{K}_{\mathrm{SV}}$ values were obtained as tabulated in Table 1 . In dynamic quenching, $10^{10} / \mathrm{M} \cdot \mathrm{s}$ is the maximum value for the quenching constant in the scatter collision of various quenchers and biopolymers [Lakowicz \& Weber, 1973]. Principally, fluorescence quenching depends on temperature changes, and thus, the main type of quenching is easily distinguished [Nair, 2018]. For the static quenching mechanism, $\mathrm{K}_{\mathrm{SV}}$ values decreased with increasing temperature, but the opposite results are expected for dynamic quenching. As shown in Table $1, \mathrm{~K}_{\mathrm{SV}}$ and $\mathrm{K}_{\mathrm{q}}$ values clearly decreased as the temperature increased. Additionally, the calculated values for $\mathrm{K}_{\mathrm{q}}$ are much larger than $10^{10} / \mathrm{M} \cdot \mathrm{s}$, indicating that the HSA quenching mechanism induced by CA is static quenching and not dy-
TABLE 1. Stern-Volmer quenching constant $\left(\mathrm{K}_{\mathrm{SV}}\right)$ and a bimolecular quenching rate constant $\left(\mathrm{K}_{\mathrm{q}}\right)$ for the binding of caffeic acid to human serum albumin at different temperatures.

\begin{tabular}{lccc}
\hline $\mathrm{T}(\mathrm{K})$ & $\mathrm{K}_{\mathrm{SV}}\left(\times 10^{4} / \mathrm{M}\right)$ & $\mathrm{K}_{\mathrm{q}}\left(\times 10^{12} / \mathrm{M} \cdot \mathrm{s}\right)$ & $\mathrm{r}^{\mathrm{a}}$ \\
\hline 290 & 4.25 & 4.25 & 0.9997 \\
300 & 2.43 & 2.43 & 0.9907 \\
310 & 1.53 & 1.53 & 0.9949 \\
\hline
\end{tabular}

${ }^{a} \mathrm{r}$ is the linear correlation coefficient.

TABLE 2. Modified Stern-Volmer association constant $\left(\mathrm{K}_{\mathrm{a}}\right)$ for the caffeic acid-human serum albumin (CA-HSA) interaction at different temperatures $(T)$ and the values for enthalpy $(\Delta H)$, entropy $(\Delta S)$, and Gibb's free energy $(\Delta \mathrm{G})$ which were calculated as thermodynamic parameters for the binding of CA to HSA.

\begin{tabular}{l|c|c|c|c|c}
\hline $\mathrm{T}(\mathrm{K})$ & $\begin{array}{c}\mathrm{K}_{\mathrm{a}} \\
\left(\times 10^{4} / \mathrm{M}\right)\end{array}$ & $\mathrm{r}^{\mathrm{a}}$ & $\begin{array}{c}\Delta \mathrm{H} \\
(\mathrm{kJ} / \mathrm{mol})\end{array}$ & $\begin{array}{c}\Delta \mathrm{S} \\
(\mathrm{J} / \mathrm{mol} \cdot \mathrm{K})\end{array}$ & $\begin{array}{c}\Delta \mathrm{G} \\
(\mathrm{kJ} / \mathrm{mol})\end{array}$ \\
\hline 290 & 5.85 & 0.9992 & & & -26.43 \\
300 & 3.52 & 0.9992 & -32.75 & -21.78 & -26.22 \\
310 & 2.44 & 0.9996 & & & -26.00
\end{tabular}

${ }^{a} \mathrm{r}$ is the linear correlation coefficient.

TABLE 3. Binding constants $\left(K_{b}\right)$ and the number of binding sites (n) for the interaction of caffeic acid with human serum albumin at different temperatures $(\mathrm{T})$.

\begin{tabular}{lcccc}
\hline $\mathrm{T}(\mathrm{K})$ & $\mathrm{K}_{\mathrm{b}}\left(\times 10^{4} / \mathrm{M}\right)$ & $\mathrm{n}$ & $\mathrm{r}^{\mathrm{a}}$ \\
\hline 290 & 2.18 & 0.93 & 0.9981 \\
300 & 1.14 & 0.92 & 0.9940 \\
310 & 0.64 & 0.91 & 0.9962 \\
\hline
\end{tabular}

${ }^{a} \mathrm{r}$ is the linear correlation coefficient.

namic quenching. Also other phenolic acids (cinnamic acid), flavonoids (glabridin, diosmetin), and stilbenes (resveratrol) quenched the fluorescence of HSA through the static mode [Nair, 2018; Razzak et al., 2019; Sun et al., 2018].

As observed in Figure $1 \mathrm{C}$, plotting $\mathrm{F}_{0} /\left(\mathrm{F}_{0}-\mathrm{F}\right)$ vs. $1 /[\mathrm{Q}]$ yields $1 / f_{a}$ and $1 /\left(f_{a} K_{a}\right)$ as the $y$-coordinate and the slope, respectively. Table 2 lists the calculated $\mathrm{K}_{\mathrm{a}}$ values for the CA-HSA complex. Similarly, as the temperature increased, the obtained $\mathrm{K}_{\mathrm{a}}$ values showed a decreasing trend verifying the decreasing trend observed for the $\mathrm{K}_{\mathrm{SV}}$ (Table 1).

\section{Binding constant and the number of binding sites}

Figure 1D displays the $\log \left[\left(\mathrm{F}_{0}-\mathrm{F}\right) / \mathrm{F}\right]$ vs. $\log [\mathrm{Q}]$ plots of the CA-HSA complex investigated at the three temperatures, and Table 3 summarizes the corresponding values calculated for $n$ and $\mathrm{K}_{\mathrm{b}}$. An increase in temperature led to a decrease in the values of $\mathrm{K}_{\mathrm{b}}$. The CA-HSA complex was not stable as the temperature increased, which might explain the decreasing values of $\mathrm{K}_{b}$. According to the obtained values for the number of binding sites, $n$ values were near unity, indicating that CA had one independent binding site on the HSA 
molecule. The results obtained here were in good agreement with the values reported before. For example, comparative studies on the interaction of chlorogenic acid, caffeic acid, and ferulic acid with bovine serum albumin (BSA) using UV absorption spectroscopy, fluorescence spectroscopy, and synchronous fluorescence spectroscopy showed that the binding constant and $\mathrm{n}$ values were in the order of $10^{4} / \mathrm{M}$ and $\sim 1$, respectively [Li et al., 2010]. Similar results were also reported for CA when the interactions between different phenolics and BSA were investigated using fluorescence quenching and molecular docking [Skrt et al., 2012].

\section{UV-Vis spectroscopy}

The structural changes and an understanding of the formation of a complex between different molecules and proteins are usually investigated with a suitable and effortless spectroscopic technique, such as UV-Vis spectroscopy. Therefore, UV-Vis spectra were recorded, and the results are shown in Figure 1F. At the wavelength of $280 \mathrm{~nm}$, the intensity of the recorded UV-Vis spectra of HSA increased when increasing concentrations of CA were applied. The position of the maxima was also slightly shifted to higher wavelengths. These observations might be related to the change in the polarity and the hydrophobicity around the Trp residue [Ulrich, 1981, 1990; Tao et al., 1981], as observed in the fluorescence experiments. Hence, CA binds HSA and induces conformational changes in the albumin structure [Cui et al., 2004]. The transfer of energy during the collision between albumin molecules and the interacting substances is mainly mediated by dynamic quenching, with no alterations in the UV-Vis spectrum of albumin. However, for static quenching, the formation of complexes between various chemicals and proteins causes a decrease or an increase in the intensity of the UV-Vis spectrum for albumin [Jahanban-Esfahlan et al., 2017; Roufegarinejad et al., 2019]. In the present study, HSA fluorescence emission quenching was principally related to the formation of the CA-HSA complex. Thus, the results of fluorescence quenching obtained for CA-HSA were sufficiently supported by the changes in the UV-Vis spectra.

\section{Thermodynamic parameters}

Typically, vdW forces, electrostatic forces, H-bonding, and hydrophobic interactions are the key forces driving the interaction of various chemicals and biomacromolecules [Bourassa et al., 2011; Dan et al., 2019, Nair, 2018; Zou et al., 2019]. The changes in Gibb's free energy $(\Delta G)$, enthalpy $(\Delta \mathrm{H})$, and entropy $(\Delta \mathrm{S})$, the main thermodynamic parameters, should be considered to obtain a comprehensive understanding of the complexation mode between different ligands and proteins.

The process underlying the CA and HSA interaction was spontaneous because the value obtained for $\Delta \mathrm{G}$ was negative, from -26.43 to $-26.00 \mathrm{~kJ} / \mathrm{mol}$ (Table 2). In proteinligand interactions and according to the studies by Ross \& Subramanian [1981], the amounts and the sign of thermodynamic parameters $(\Delta \mathrm{S}$ and $\Delta \mathrm{H})$ are useful for studying the contribution of the main forces to the stability of the ligand-protein complexes. From the thermodynamic perspec- tive, $\Delta \mathrm{H}<0$ and $\Delta \mathrm{S}>0$ represent support electrostatic forces, $\Delta \mathrm{H}$ and $\Delta \mathrm{S}>0$ suggest hydrophobic interactions, and $\Delta \mathrm{S}$ and $\Delta \mathrm{H}<0$ represent the $\mathrm{H}$-bonding and $\mathrm{vdW}$ forces. As presented in Table 2, the calculated values for $\Delta \mathrm{S}$ and $\Delta \mathrm{H}$ in this study were $-21.78 \mathrm{~kJ} / \mathrm{mol}$ and $-32.75 \mathrm{~J} / \mathrm{mol} \cdot \mathrm{K}$, respectively. The values calculated for $\Delta \mathrm{S}$ and $\Delta \mathrm{H}$ were negative indicating the enthalpy-driven interaction of CA and HSA; however, the entropy was unfavourable. Accordingly, the main driving forces stabilizing the complex were $\mathrm{H}$-bonding and $\mathrm{vdW}$ forces.

\section{Energy transfer}

Forster resonance energy transfer (FRET) is known as the interaction between molecules with electronically excited states without the emission of a photon. The phenomenon is distance-dependent, and the energy of excitation is transferred from one donor molecule to another acceptor molecule [Lakowicz, 2006]. Several factors affect the FRET efficiency, such as: (1) the overlap region of the acceptor UV-Vis spectra and the donor emission, (2) the transition dipole orientation of the donor and acceptor, and (3) the distance between the donor and acceptor. The overlap in the spectroscopic region between the UV-Vis absorption spectrum of CA and the emission spectrum of HSA fluorescence is shown in Figure 1A.

Using equations (7), (8), and (9), $\mathrm{J}=1.6637 \times 10^{-19} \mathrm{~cm}^{3} / \mathrm{M}$, $\mathrm{E}=0.075, \mathrm{R}_{0}=3.6616 \mathrm{~nm}$, and $\mathrm{r}=1.92 \mathrm{~nm}$ were calculated for the CA-HSA complex. Here, the calculated value of $r$ for the interaction of CA with HSA was consistent with the value reported in a previous study [Li et al., 2010]. The probability of energy transfer is high when the average distance of the acceptor and donor molecules is less than $8 \mathrm{~nm}$ [Samari et al., 2012]. The calculated value for the distance between the donor molecule (Trp residue) in HSA and the acceptor molecules (the interacting CA) was approximately $2 \mathrm{~nm}$. Additionally, the calculated values for $r$ and $R_{0}$ followed the rule $0.5 \mathrm{R}_{0}<\mathrm{r}<1.5 \mathrm{R}_{0}$ [Jahanban-Esfahlan et al., 2017; Roufegarinejad et al., 2019.] Thus, energy was transferred during the CA and HSA interaction.

\section{Molecular docking}

CA was docked to HSA using ArgusLab software to determine the ideal binding site and the binding mode. Argus$\mathrm{Lab}$ is a useful docking program that performs computational molecular docking to provide researches an understanding of the interaction of different molecules with albumin. The best conformational binding mode for the interaction of CA and HSA is displayed in Figure 2B. CA binds to another site of the HSA molecule that differs from the known binding sites I and II. CA interacts with HSA within subdomain IA in domain I. Figure $2 \mathrm{C}$ shows the $\mathrm{H}$-bonds that formed between the CA molecule and the amino acids of HSA. The amino acid residues Asn-9, Gly-248, Asp-249, Leu-251, and Glu-252 formed H-bonds with the CA molecule. Moreover, six other amino acid residues of HSA, including Tyr-30, Phe-102, Gly-71, Leu-250, His-67 and Leu-74, surrounded the CA molecule in its binding site (these amino acids are not shown in the figure). The binding energy for CA-HSA complex was determined to be $-9.75 \mathrm{kcal} / \mathrm{mol}$ from the docking calculations. Both hydrophobic and hydrophilic 
A

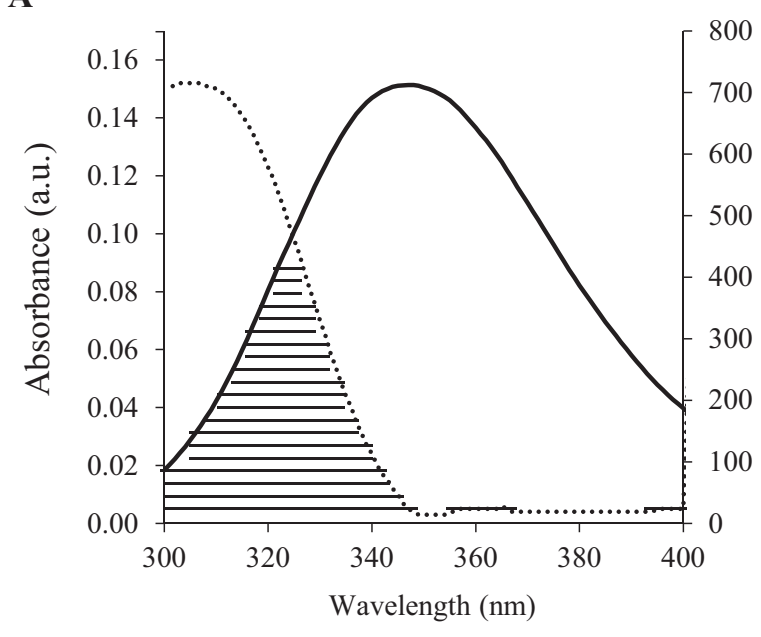

B

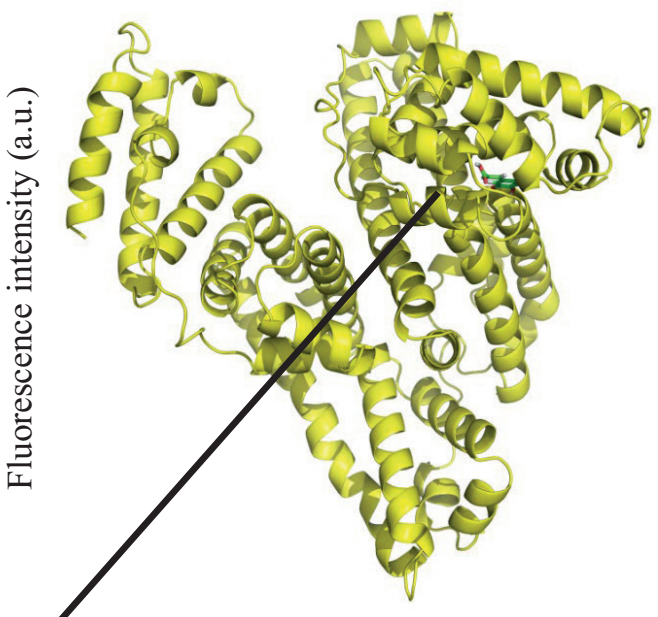

C

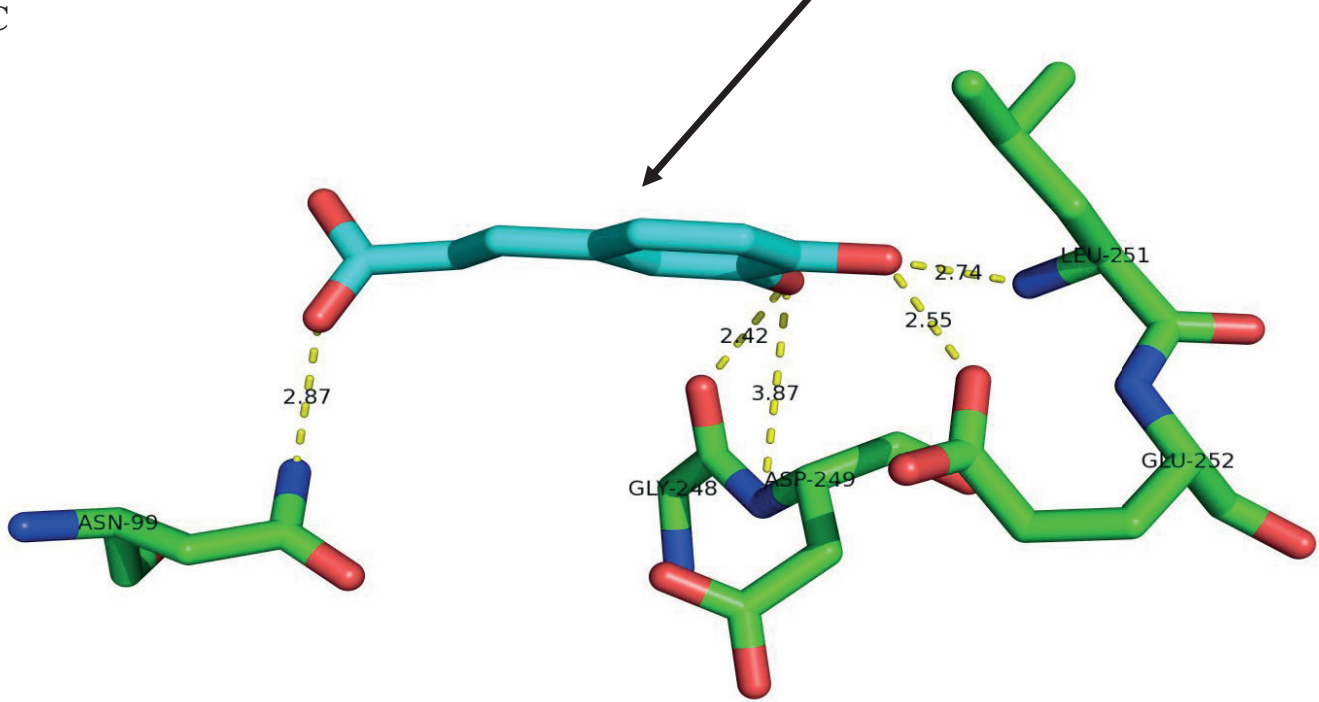

FIGURE 2. The overlap between the UV-Vis absorption spectrum of caffeic acid (CA) with human serum albumin (HSA) fluorescence emission spectrum at $25^{\circ} \mathrm{C}$. The concentrations of CA and HSA were $30 \mu \mathrm{M}$. The spectrum of CA is shown as a solid dark line, and the spectrum of HSA is depicted as the dotted line. The overlap between the CA and HSA spectra is presented as the shaded part (A). CA docked with HSA, as illustrated in a cartoon image. CA and HSA molecules are represented in stick mode and yellow cartoon models, respectively (B). H-bonds between HSA amino acids and the docked CA molecule. Yellow dashed lines show H-bonds and the corresponding distances. The CA structure and amino acids are displayed in stick mode. Hydrogen atoms are not shown (C).

amino acids were located in the binding site of CA. Finally, molecular docking studies revealed the formation of numerous H-bonds in the CA-HSA confirming the results of fluorescence experiments.

\section{CONCLUSIONS}

In the present work, the interactions between CA and HSA have been analyzed using fluorescence spectroscopy, UV-Vis spectroscopy, and molecular docking methods, and numerous binding parameters have been obtained. A static quenching mechanism was identified for the intrinsic quenching of the HSA fluorescence by CA. The obtained values for the number of binding sites showed the presence of a single class of binding sites for the CA molecule on HSA. The interaction of CA and HSA was determined to be enthalpydriven and spontaneous. The main driving forces stabilizing the complex were $\mathrm{H}$-bonds and $\mathrm{vdW}$ forces. The molecular docking calculations indicated that CA binds to HSA in subdomain IA of domain I of HAS, and the detected H-bonds confirmed the results of fluorescence spectroscopy. According to the results of fluorescence and UV spectroscopy, CA induced conformational changes in the albumin structure.

Caffeic acid shows a high affinity for albumin, and thus this phenolic compound would be distributed in the body upon interacting with HSA.

\section{CONFLICT OF INTERESTS}

Authors declare no conflict of interests.

\section{ORCID IDs}

R. Amarowicz https://orcid.org/0000-0001-9731-0045

A. Jahanban-Esfahlan https://orcid.org/0000-0001-8693-3837 J.M. Lorenzo https://orcid.org/0000-0002-7725-9294 


\section{REFERENCES}

1. Adzet, T., Camarasa, J., Escubedo, E., Merlos, M. (1988). In vitro study of caffeic acid - bovine serum albumin interaction. European Journal of Drug Metabolism and Pharmacokinetics, 13(1), $11-14$.

https://doi.org/10.1007/BF03189921

2. Belatik, A., Hotchandani, S., Bariyanga, J., Tajmir-Riahi, H. (2012). Binding sites of retinol and retinoic acid with serum albumins. European Journal of Medicinal Chemistry, 48, 114-123. https://doi.org/10.1016/j.ejmech.2011.12.002

3. Bhat, S., Azmi, A., Hadi, S. (2007). Prooxidant DNA breakage induced by caffeic acid in human peripheral lymphocytes: Involvement of endogenous copper and a putative mechanism for anticancer properties. Toxicology and Applied Pharmacology, 218(3), 249-255.

https://doi.org/10.1016/j.taap.2006.11.022

4. Bourassa, P., Hasni, I., Tajmir-Riahi, H. (2011). Folic acid complexes with human and bovine serum albumins. Food Chemistry, 129(3), 1148-1155.

https://doi.org/10.1016/j.foodchem.2011.05.094

5. Chen, G.-Z., Huang, X.-Z., Xu, J.-G., Zheng, Z., Wang, Z. (Eds). (1990). The Methods of Fluorescence Analysis. $2^{\text {nd }}$ edition. Science Press, Beijing, pp.112-117.

6. Chen, J.H., Ho, C.-T. (1997). Antioxidant activities of caffeic acid and its related hydroxycinnamic acid compounds. Journal of Agricultural and Food Chemistry, 45(7), 2374-2378. https://doi.org/10.1021/jf970055t

7. Chung, M., Walker, P., Hogstrand, C. (2006). Dietary phenolic antioxidants, caffeic acid and Trolox, protect rainbow trout gill cells from nitric oxide-induced apoptosis. Aquatic Toxicology, 80(4), 321-328.

https://doi.org/10.1016/j.aquatox.2006.09.009

8. Cui, F.-L., Fan, J., Li, J.-P., Hu, Z.-D. (2004). Interactions between 1-benzoyl-4-p-chlorophenyl thiosemicarbazide and serum albumin: investigation by fluorescence spectroscopy. Bioorganic \& Medicinal Chemistry, 12(1), 151-157.

https://doi.org/10.1016/j.bmc.2003.10.018

9. Dan, Q., Xiong, W., Liang, H., Wu, D., Zhan, F., Chen, Y., Ding, Li, B. (2019). Characteristic of interaction mechanism between $\beta$-lactoglobulin and nobiletin: A multi-spectroscopic, thermodynamics methods and docking study. Food Research International, 120, 255-263.

https://doi.org/10.1016/j.foodres.2019.01.003

10. Eftink, M.R. (1991). Fluorescence quenching reactions: Probing biological macromolecular structures. In T.G. Dewey (Ed.). Biophysical and Biochemical Aspects of Fluorescence Spectroscopy. $1^{\text {st }}$ edition, Springer Science + Business Media LCC, New York, USA, chapter 1, pp. 1-42.

https://doi.org/10.1007/978-1-4757-9513-4

11. El-Seedi, H.R., El-Said, A.M.A. Khalifa, S.A.M., Göransson, U., Bohlin, L., Borg-Karlson, A.-K., Verpoorte, R. (2012). Biosynthesis, natural sources, dietary intake, pharmacokinetic properties, and biological activities of hydroxycinnamic acids. Journal of Agricultural and Food Chemistry, 60(44), 10877-10895. https://doi.org/10.1021/jf301807g

12. Jahanban-Esfahlan, A., Dastmalchi, S., Davaran, S. (2016). A simple improved desolvation method for the rapid preparation of albumin nanoparticles. International Journal of Biomolecular Macromolecules, 91, 703-709.

https://doi.org/10.1016/j.ijbiomac.2016.05.032

13. Jahanban-Esfahlan, A., Davaran, S., Moosavi-Movahedi, A.A., Dastmalchi, S. (2017). Investigating the interaction of juglone (5-hydroxy-1,4-naphthoquinone) with serum albumins using spectroscopic and in silico methods. Journal of the Iranian Chemical Society, 14, 1527-1540.

https://doi.org/10.1007/s13738-017-1094-0

14. Jahanban-Esfahlan, A., Ostadrahimi, A., Jahanban-Esfahlan, R., Roufegarinejad, L., Tabibiazar, M., Amarowicz, R. (2019). Recent developments in the detection of bovine serum albumin. International Journal of Biomolecular Macromolecules, 138, 602-617.

https://doi.org/10.1016/j.ijbiomac.2019.07.096

15. Jahanban-Esfahlan, A., Panahi-Azar, V., Sajedi, S. (2015). Spectroscopic and molecular docking studies on the interaction between $\mathrm{N}$-acetyl cysteine and bovine serum albumin. Biopolymers, 103, 638-645.

https://doi.org/10.1002/bip.22697

16. Jahanban-Esfahlan, A., Roufegarinejad, L., Jahanban-Esfahlan, R., Tabibiazar, M., Amarowicz, R. (2020). Latest developments in the detection and separation of bovine serum albumin using molecularly imprinted polymers. Talanta, 207, art. no. 120317.

https://doi.org/10.1016/j.talanta.2019.120317

17. Lakowicz, J.R. (2006). Principles of Fluorescence Spectroscopy. $3^{\text {rd }}$ edition, Springer Science + Business Media, New York, USA, chapter 13, pp. 443-452.

https://doi.org/10.1007/978-0-387-46312-4

18. Lakowicz, J.R., Weber, G. (1973). Quenching of fluorescence by oxygen. Probe for structural fluctuations in macromolecules. Biochemistry, 12(21), 4161-4170.

https://doi.org/10.1021/bi00745a020

19. Lehrer, S. (1971). Solute perturbation of protein fluorescence. Quenching of the tryptophyl fluorescence of model compounds and of lysozyme by iodide ion. Biochemistry, 10(17), 3254-3263. https://doi.org/10.1021/bi00793a015

20. Li, S., Huang, K., Zhong, M., Guo, J., Wang, W.-Z., Zhu, R. (2010). Comparative studies on the interaction of caffeic acid, chlorogenic acid and ferulic acid with bovine serum albumin. Spectrochimica Acta, Part A: Molecular and Biomolecular Spectroscopy, 77(3), 680-686.

https://doi.org/10.1016/j.saa.2010.04.026

21. Min, J., Meng-Xia, X., Dong, Z., Yuan, L., Xiao-Yu, L., Xing, C. (2004). Spectroscopic studies on the interaction of cinnamic acid and its hydroxyl derivatives with human serum albumin. Journal of Molecular Structures, 692(1-3), 71-80.

https://doi.org/10.1016/j.molstruc.2004.01.003

22. Mrkalić, E., Jelićb, R., Stojanović, S., Sovrlić, M. (2021). Interaction between olanzapine and human serum albumin and effect of metal ions, caffeine and flavonoids on the binding: A spectroscopic study. Spectrochimica Acta Part A: Molecular and Biomolecular Spectroscopy, 249, art. no. 119295. https://doi.org/10.1016/j.saa.2020.119295

23. Nair, M.S. (2018). Spectroscopic studies on the interaction of serum albumins with plant derived natural molecules. Applied Spectroscopy Reviews, 53(8), 636-666.

https://doi.org/10.1080/05704928.2017.1402184 
24. Olthof, M.R., Hollman, P. C.-H., Katan, M.B. (2001). Chlorogenic acid and caffeic acid are absorbed in humans. The Journal of Nutrition, 131(1), 66-71.

https://doi.org/10.1093/jn/131.1.66

25. Pirjo, M., Hellström, J., Törrönen, R. (2006). Phenolic acids in berries, fruits, and beverages. Journal of Agricultural and Food Chemistry, 53(19), 7193-7199.

https://doi.org/10.1021/jf0615247

26. Precupas, A., Sandu, R., Cantemir, A.R., Anghel, D.-F., Popa, V.T. (2017). Interaction of caffeic acid with bovine serum albumin is complex: Calorimetric, spectroscopic and molecular docking evidence. New Journal of Chemistry, 41, 15003-15015. https://doi.org/10.1039/C7NJ03410E

27. Rashmi, H.B., Negi, P.S. (2020). Phenolic acids from vegetables: A review on processing stability and health benefits. Food Research International, 136, art. no. 109298. https://doi.org/10.1016/j.foodres.2020.109298

28. Razzak M.A., Lee, J.-E., Choi, S.S. (2019). Structural insights into the binding behavior of isoflavonoid glabridin with human serum albumin. Food Hydrocolloids, 91, 290-300. https://doi.org/10.1016/j.foodhyd.2019.01.031

29. Ross, P.D., Subramanian, S. (1981). Thermodynamics of protein association reactions: forces contributing to stability. Biochemistry, 20(11), 3096-3102.

https://doi.org/10.1021/bi00514a017

30. Roufegarinejad, L., Amarowicz, R., Jahanban-Esfahlan, A. (2019). Characterizing the interaction between pyrogallol and human serum albumin by spectroscopic and molecular docking methods. Journal of Biomolecular Structure and Dynamics, 37(11), 2766-2775.

https://doi.org/10.1080/07391102.2018.1496854

31. Samari, F., Shamsipur, M., Hemmateenejad, B., Khayamian, T., Gharaghani, S. (2012). Investigation of the interaction between amodiaquine and human serum albumin by fluorescence spectroscopy and molecular modeling. European Journal of Medicinal Chemistry, 54, 255-263.

https://doi.org/10.1016/j.ejmech.2012.05.007

32. Sinisi, V., Forzato, C., Cefarin, N., Navarini, L., Berti, F. (2015). Interaction of chlorogenic acids and quinides from coffee with human serum albumin. Food Chemistry, 168, 332-340. https://doi.org/10.1016/j.foodchem.2014.07.080

33. Skrt, M., Benedik, E., Podlipnik, C., Ulrih, N.P. (2012). Interactions of different polyphenols with bovine serum albumin using fluorescence quenching and molecular docking. Food Chemistry, $135,2418-2424$.

https://doi.org/10.1016/j.foodchem.2012.06.114

34. Sova, M., Saso, L. (2020). Natural sources, pharmacokinetics, biological activities and health benefits of hydroxycinnamic acids and their metabolites. Nutrients, 12(8), art. no. 2190.

https://doi.org/10.3390/nu12082190
35. Sułkowska, A. (2002). Interaction of drugs with bovine and human serum albumin. Journal of Molecular Structure, 614(1-3), 227-232.

https://doi.org/10.1016/S0022-2860(02)00256-9

36. Suryaprakash, P., Kumar, R.P., Prakash, V. (2000). Thermodynamics of interaction of caffeic acid and quinic acid with multisubunit proteins. International Journal of Biological Macromolecules, 27(3), 219-228.

https://doi.org/10.1016/S0141-8130(00)00119-7

37. Sun, Q., Yang, H., Tang, P., Liu, J., Wang, W., Li, H. (2018). Interactions of cinnamaldehyde and its metabolite cinnamic acid with human serum albumin and interference of other food additives. Food Chemistry, 243, 74-81.

https://doi.org/10.1016/j.foodchem.2017.09.109

38. Sudhamalla, B., Gokara, M., Ahalawat, N., Amooru, D.G., Subramanyam, R. (2010). Molecular dynamics simulation and binding studies of $\beta$-sitosterol with human serum albumin and its biological relevance. Journal of Physical Chemistry, 114(27), 9054-9062.

https://doi.org/10.1021/jp102730p

39. Tomašević, M., Lisjak, K., Vanzo, A., Ganić, K.K. (2019). Changes in the composition of aroma and phenolic compounds induced by different enological practices of Croatian white wine. Polish Journal of Food and Nutrition Sciences, 69(4), 343-358. https://doi.org/10.31883/pjfns/112328

40. Ulrich, K.-H. (1981). Molecular aspects of ligand binding to serum albumin. Pharmacological Reviews, 33(1), 17-53.

41. Ulrich, K.-H. (1990). Structure and ligand binding properties of human serum albumin. Danish Medical Bulletin, 37(1), 57-84.

42. Wang, N., Ye, L., Yan, F., Xu, R. (2008). Spectroscopic studies on the interaction of azelnidipine with bovine serum albumin. International Journal of Pharmaceutics, 351 (1-2), 55-60. https://doi.org/10.1016/j.ijpharm.2007.09.016

43. Worldwide Protein Data Bank (wwPDB). Available online: [https://www.rcsb.org/structure/1ao6] (accessed on 10 April 2020).

44. Zhang, Y., Yue, Y., Li, J., Chen, X. (2008). Studies on the interaction of caffeic acid with human serum albumin in membrane mimetic environments. Journal of Photochemistry and Photobiology B: Biology, 90(3), 141-151. https://doi.org/10.1016/j.jphotobiol.2007.12.004

45. Zou, Y.-C., Wu, C.-L., Ma, C.-F., He, S., Brennan, C.S., Yuan, Y. (2019). Interactions of grape seed procyanidins with soy protein isolate: Contributing antioxidant and stability properties. $L W T-$ Food Science and Technology, 115, art. no. 108465. https://doi.org/10.1016/j.Iwt.2019.108465

Submitted: 10 December 2020. Revised: 8 February 2021. Accepted: 10 February 2021. Published on-line: 24 February 2021. 